\title{
Research on Global Path Planning in the Marine Environment for AUV
}

\author{
Rubo Zhang Chunhui Xu Ziying Zhang Dong Xu \\ College of Computer Science and Technology, Harbin Engineering University, Harbin 150001, P. R. China
}

\begin{abstract}
This paper presents a saving energy consumption approach to the AUV global path planning based GA. The AUV works in the large-scale marine environments and the energy consumption is a big problem when the AUV is working. Ocean current is a main factor that impacts AUV's navigation. We consider ocean current as an evaluation factor, and make it a part of fitness function in GA. We take ocean current factor into account in global path planning.
\end{abstract}

Keywords: AUV, Global path planning, Ocean current, GA

\section{Introduction}

Path planning is a classical problem in AI, and it's also an important part of mobile robot navigation. Genetic algorithm (GA) is a global optimization algorithm based on natural selection and natural genetic. It utilizes operators such as selection operator, crossover operator and mutation operator, which are abstracted from natural selection and genetic operator. GA is an iterative procedure consisting of a constant-size population of individuals, and each of them is represented by a finite string of symbols, known as the genome, encoding a possible solution in a given problem space. This space, referred to as the search space, comprises all possible solutions to the problem at hand. GA has a number of advantages. It can quickly scan a vast solution set, the viable solutions exchange information by crossover and mutation to create new solutions, not trapped in local minima. It doesn't have to know any rules of the problem, and it works by its own internal rules. So GA only need the value of the feasible solutions, doesn't care about the continuity of objective function. It uses probability to select and initialize solution, so it's well adaptive and robust. This is very useful for complex or loosely defined problems.

AUVs usually work in marine environments characterized by complex spatial variability. This spatial complexity is induced by the turbulent nature of the ocean, described by the continuous change of a wide range of spatial and time scales. Ocean current is a kind of energetic flows which can strongly perturb the development of AUVs operations. In particular, AUVs usually encounter strong current fields in the marine environment that can jeopardize their missions. So the navigation must consider the impact of ocean current. Determining and predicting ocean currents is then a fundamental requirement to plan an optimal path, specially, the AUV works in the large-scale marine environment, it has to consider the energyexhaustive. The AUV should take full advantage of the energetic field of ocean current, along the direction of ocean current and avoid energy consumption for resistance to current as possible as it can [1].

This paper is organized as follows. Section 2 constructs the working environment of the AUV, including working space and ocean current. Section 3 describes the GA based approach to global path planning in 2-D, and Section 4 shows the simulation and experimental results in 2-D. Section 5 describes the approach to 3-D layered path planning, then gives the experimental results. Section 6 gives the conclusion.

\section{Environmental modeling}

AUV's working space is three-dimension marine environment in fact. Divide the working space into layers by depth, each layer is a 2-D x-y plane coordinate system. The $x-y$ coordinate is separated into grids. Each grid is a square, and assuming the length of the grid is $20 \mathrm{~km}$. Any point of the grid defines a node $x=(h, k), 0 \leq h<m, 0 \leq k<n$.

Ocean current is a vector field, and each point is described with value and direction. Assume the ocean currents in the same grid are identical. We utilize stream function to simulate ocean current of AUV's working space. The mathematical model [2] expression is:

$$
\psi(x, y)=1-\tanh \left(\frac{y-B(t) \cos (k(x-c t))}{\left(1+k^{2} B(t)^{2} \sin ^{2}(k(x-c t))\right)^{\frac{1}{2}}}\right)
$$


where $B(t)=b+e \cos (\omega t+\theta), b=1.2, c=0.12, k=$ 0.84, $\omega=0.4, e=0.3, \theta=\pi / 2$.

$$
U(x, y, t)=-\frac{\partial \psi}{\partial y} \quad V(x, y, t)=\frac{\partial \psi}{\partial x}
$$

Where $U(x, y, t), V(x, y, t)$ are, respectively, $\mathrm{x}$ axis and $y$ axis components of the ocean current field at time $t$ in the position $(x, y)$. In this paper, $(x, y)$ is the central point in each grid (Fig. 1).

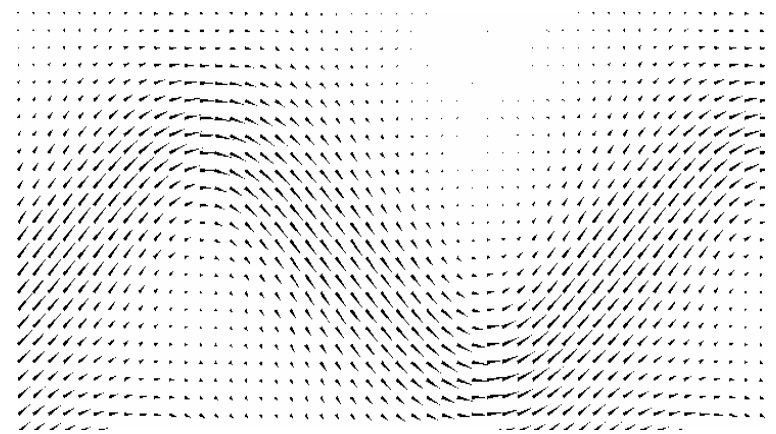

Fig. 1: Ocean Current Simulated by Stream Function

\section{GA based global path planning in 2-D}

\subsection{Path coding}

A path from start point to goal point is consisting of a series of nodes connected. Assuming the start point uses symbol $\mathrm{S}$ for short, and the goal point uses $\mathrm{G}$, path $=\left\{S, \ldots X i, X_{i+1}, \ldots, G\right\}$, each point is a node of the path, and adjacent nodes are connected with lines. Path planning problem can be summarized as follows: Given a start point, a goal point, the position of the obstacle and the ocean current. The purpose is to find a safe path with the minimum energy when the AUV moves at a constant speed. All paths will be considered strictly monotone with respect to the $\mathrm{x}$-axis so that any two adjacent nodes $X_{i}, X_{i+1}$ satisfy the relation $h_{i+1}=h_{i}+1$. Each path has nodes with fixedlength $\mathrm{m}$. Not all paths are monotone, obviously. But in the large-scale marine environment, obstacles are quite sparse, monotone paths are reasonable when the space gridding is not dense [3].

\subsection{Initialization}

The individuals of the population are generated randomly, every individual represents a path. Individual is consisting of a series of nodes from the start point to the goal point. The $\mathrm{x}$-coordinate is increased by grid interval and $y$-coordinate is generated randomly. In fact, the value of the adjacent nodes will not change greatly. So the generation of the y-coordinate is based on the adjacent node, and we restrict the $y$-coordinate generated randomly in a small range. This initialization of the individual is more reasonable.

\subsection{Fitness evaluation}

Every path need to be evaluated, energy consumption as fitness used here, which is carried out by computing and adding up the energy required to overcome the force generated by the ocean current field in each leg of the path. Consider the ith segment $X_{i-1} X_{i}$ connecting the nodes $X_{i-1}, X_{i}$ of any path. Calculate the length of this segment, and let $e_{i}$ be a unitary vector oriented along the segment $X_{i-1} X_{i}$ in the direction of the desired motion of the AUV. Consider the AUV navigates at a constant speed $c$, at any point $(x, y)$ along the segment the AUV has the actual speed $v_{i}(x, y)$ given by

$$
\begin{aligned}
& v_{i}(x, y)=c e_{i}-v_{c}(x, y) \\
& (x, y) \in X_{i-1} X_{i}
\end{aligned}
$$

Speed vector $v_{i}(x, y)$ is consisting of two components. They are $v_{x}$ and $v_{y}$ along $\mathrm{x}$ and y-axis respectively. The projection length of segment $X_{i-1} X_{i}$ along $\mathrm{x}$ and $\mathrm{y}$-axis are $d_{x}$ and $d_{y}$. The fitness function of energy consumption is fit 1 given by

$$
f i t_{1}=\sum_{X i-1 X i} \frac{1}{\sqrt{\left(v_{x} \cdot d_{x}\right)^{2}+\left(v_{y} \cdot d_{y}\right)^{2}}}
$$

We can see that the paths with the lowest energy consumption have the highest fitness values. So the higher the fitness values are, the stronger the paths are.

When there are some obstacles in the working space of the AUV, any node of the path can't cross the obstacles. According to whether the path crossing the obstacles the fitness function is given as follows:

$$
\text { fit }_{2}=\left\{\begin{array}{l}
1, \text { if no obstacle } \\
0, \text { others }
\end{array}\right.
$$

Considering the two conditions the ultimate fitness function fit is given by:

$$
\text { fit }=\text { fit }_{1} \times \text { fit }_{2}
$$

If the path cross the obstacles the fitness value is 0 , it's the worst path in the population, so it will be eliminate from the population soon. The paths with high fitness values are those take advantage of ocean current, their own energy consumptions are low.

\subsection{Definition of genetic operators}

Selection: Sort the population according with the fitness. Select $90 \%$ of the paths which are strong and the last $10 \%$ are eliminated. Substitute the last $10 \%$ paths with the best $10 \%$ paths to form new individuals. 
Crossover: Reserve the best $10 \%$ not do the crossover operation, and other individuals make pairs randomly. Each pair does 2-points crossover operation to generate their offspring. Each individual has the same $\mathrm{x}$-coordinate, crossover operator exchanges $\mathrm{y}$ coordinate of two individuals in a pair. Generate two positions $h_{1} \in(0, m-1)$ and $h_{2} \in\left(h_{1}, m\right)$ on $\mathrm{x}$ coordinate randomly, exchange $y$-coordinate that the $\mathrm{x}$-coordinate of them is between $\left(h_{1}, h_{2}\right)$.

Mutation: A small percentage of individuals do mutation operation at random. Similar with the crossover, generate mutation interval $\left(h_{1}, h_{2}\right)$, $h_{1} \in(0, m-1)$ and $h_{2} \in\left(h_{1}, m\right)$. Mutation operator changes y-coordinate randomly. In order to ensure good individual will not be lost while mutating, the best individual isn't required to participate in the mutation.

\section{Simulation and experimental results in 2-D}

The GA has been configured in such a way that the population size is 100 and the stopping criterion is given by an upper bound of 800 generations, and the mutation probability is 0.4 .

Start point: S (40,700), goal point: G $(900,700)$. After 800 generations iteration, the best path of individual is shown in Fig. 2.

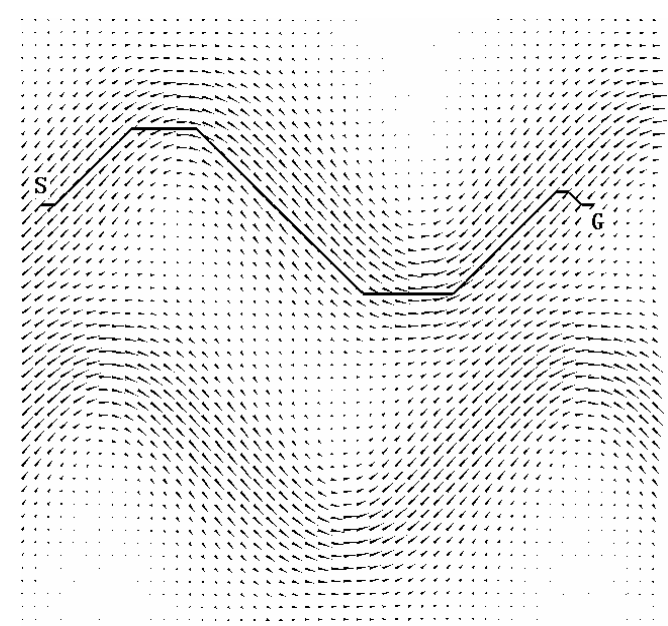

Fig. 2: Path in 2-D (a)

Start point: S $(40,500)$, goal point: G $(860,400)$. After 800 generations iteration, the best path of individual is shown in Fig. 3.

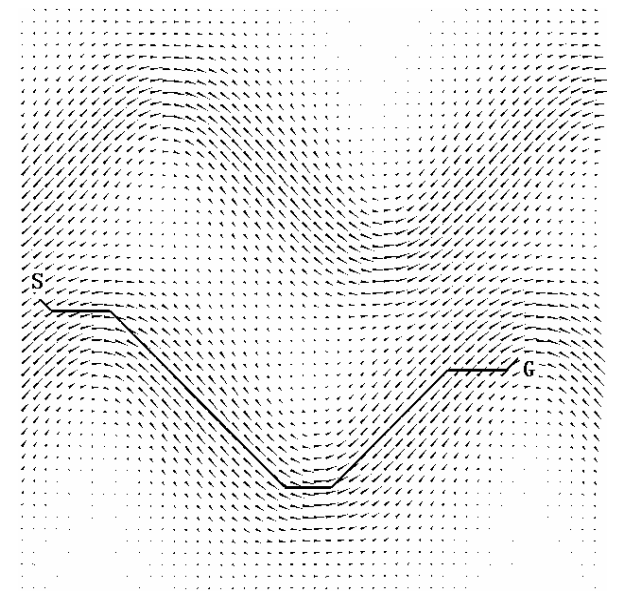

Fig. 3: Path in 2-D (b)

Then change the ocean current, using eddy current. Start point: S $(100,100)$, goal point: $G(1000,200)$. After 800 generations iteration, the best path of individual is shown in Fig. 4.

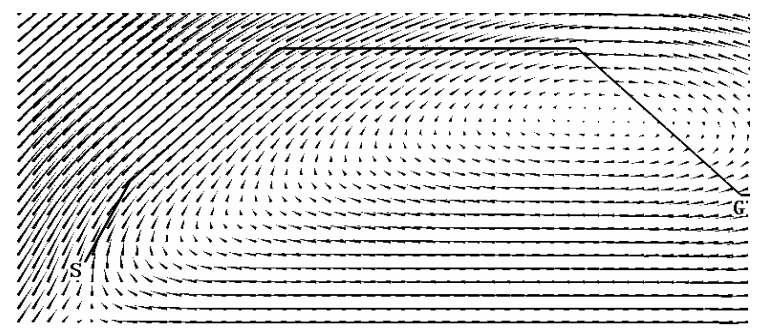

Fig. 4: Path in 2-D (c)

Start point: S $(200,200)$, goal point: $G(980,340)$. After 800 generations iteration, the best path of individual is shown in Fig. 5.

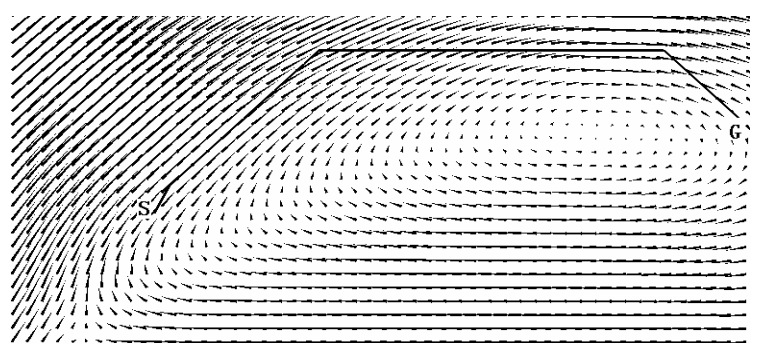

Fig. 5: Path in 2-D (d)

According to the results from the different simulations above, we can see that if we adopt the path planned by GA, which takes full advantage of the ocean current, the AUV can reduce its own energy consumption. In other words, the AUV can travel further than before using the same amount of energy. And when the ocean current is weak, the AUV tends to travel in a short path. From Fig. 4 we can see that, the ocean current is weak in the center of the eddy, the AUV can traverse the eddy to the goal point. 
Consider obstacles existing in the AUV working space, start point: S $(150,790)$, goal point: $G(850,450)$. After 800 generations iteration, the best path of individual is shown in Fig. 6.

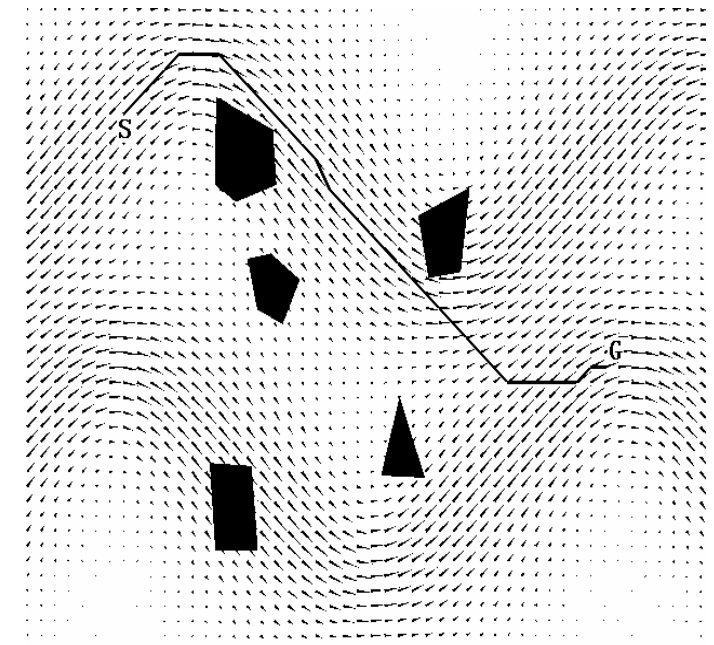

Fig. 6: Path in 2-D with Obstacles

\section{3-D layered path planning}

Marine environment is 3-D space, and the depth of the ocean is usually only hundreds of or thousands of meters, compared with its expansion, the ocean is not so deep. The AUV works in the large-scale marine environment and the global path planning in 3-D space can be considered to dividing the $3-\mathrm{D}$ space into discrete layers along the deep direction. Each layer is a 2-D space, using GA approach introduced above in 2D space to plan 2-D path in each layer. Collect some of the best path individuals of each layer together, making the initial population for 3-D. Use GA approach to generate 3-D path by changing depth between layers.

In this paper along depth we make the marine environment into four layers, the ocean current in each layer is shown in Fig. 7, they are the ocean currents in the depth of $100 \mathrm{~m}, 200 \mathrm{~m}, 300 \mathrm{~m}, 400 \mathrm{~m}$, respectively. We don't consider the impact of vertical currents.
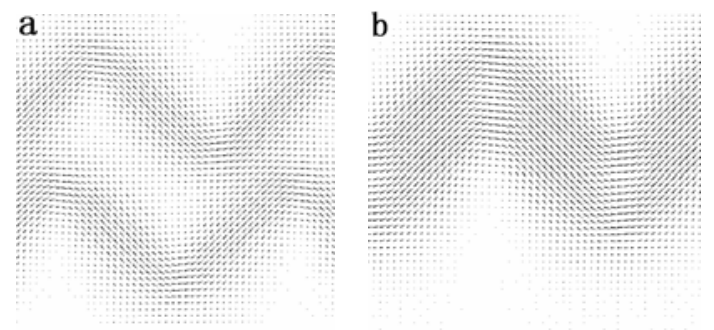
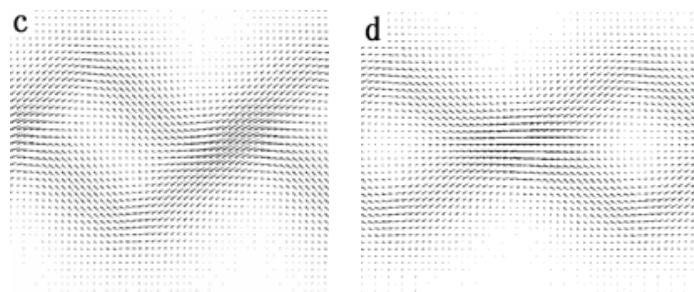

Fig. 7 :Ocean Current in Each Layer

Start point: S $(150,710,100)$, goal point: G $(810,510,400)$. Plan 2-D paths in each layer. Select 25 paths with strong fitness in each layer to generate the initial population for 3-D. After 800 generations iteration, the best path of 3-D layered is shown in Fig. 8.
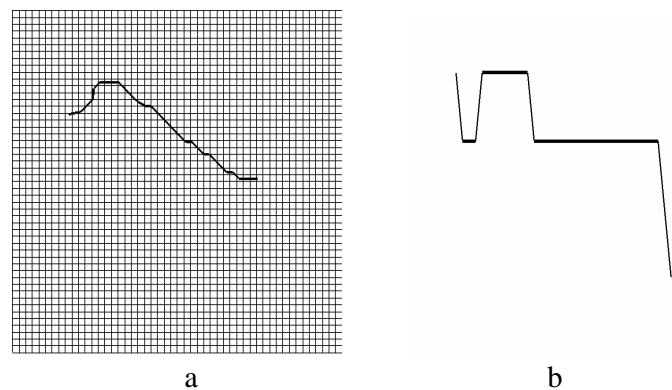

Fig.8 :3-D Path Plan by GA (a) x-y Section of the 3-D Path (b) $x$-z Section of the 3-D Path

Consider obstacles existing in the AUV working space, start point: S $(190,410,100)$, goal point: G $(850,610,400)$. The ocean current in each layer is shown in Fig. 7. The path planed by GA is shown in Fig. 9 and Fig. 10.

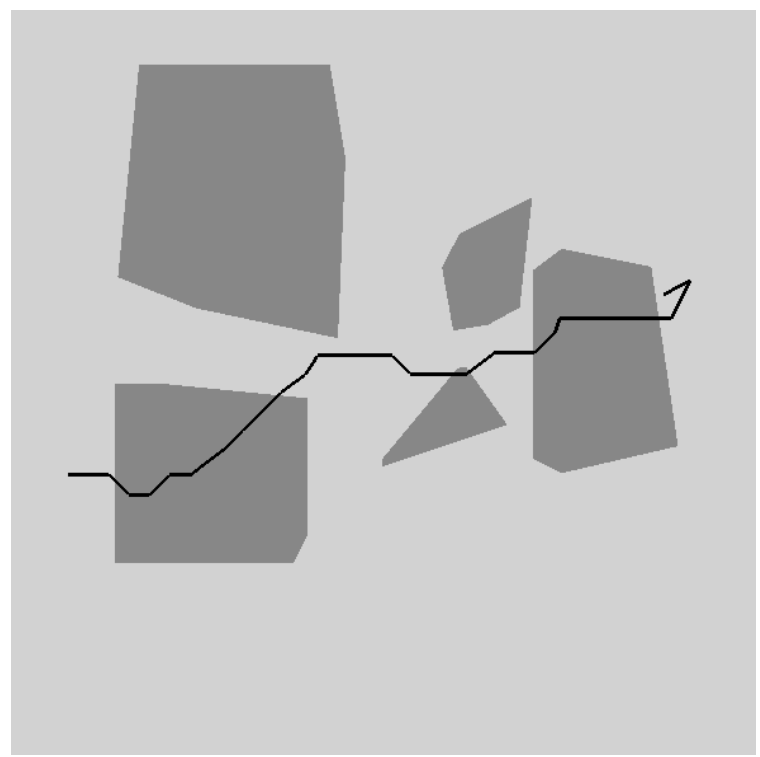

Fig. 9: Overlook the x-y Section in Simulation Environment 


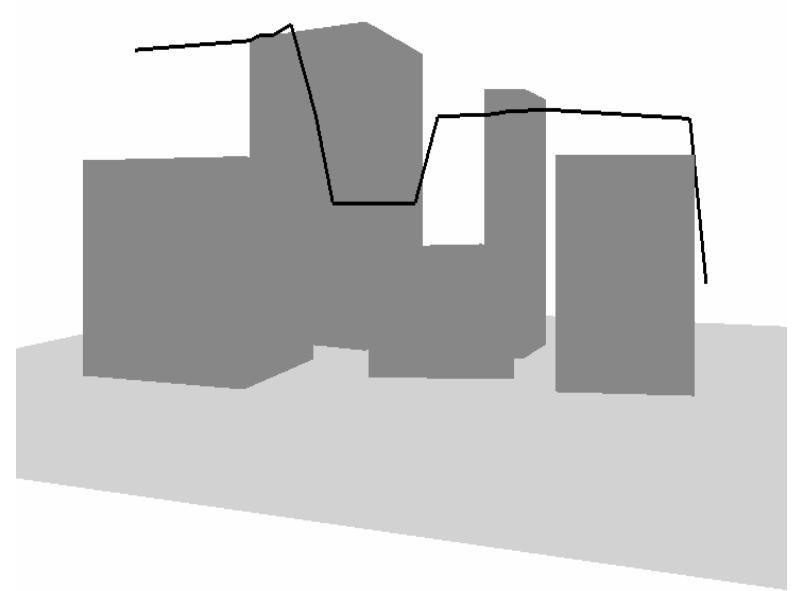

Fig. 10: Roaming in Simulation Environment

\section{Conclusions}

In this paper, we implemented the global path planning of the AUV in large-scale marine environment by using GA approach. The AUV can save its own energy by taking full advantage of ocean current. The simulation of the results in 2-D and 3-D are shown in the paper. The experiments show that the approach to planning the path is practical.

\section{References}

[1] B. Garau, A. Alvarez, Oliver G, Path planning of autonomous underwater vehicles in current fields with complex spatial variability: an A* approach. Proc. of the 2005 IEEE International Conference on Robotics and Automation, pp.195-199,2005.

[2] A. Alvarez, A. Caiti, R. Onken, Evolutionary path planning for autonomous underwater vehicles in a variable ocean. IEEE Journal of Oceanic Engineering, 29:418-423, 2004.

[3] K. Sugihara , J. Yuh, GA-based motion planning for underwater robotic vehicle, Proc. 10th Int. Symp. Unmanned Untethered Submersible Technology, pp.406-415, 1997. 